\section{Takotsubo syndrome without major stress mimicking myocarditis}

\author{
Sepideh Taghavi, (D) Maryam Chenaghlou, \\ (D) Marzieh Mirtajaddini', (D) Nasim Naderi, (D) Ahmad Amin \\ Department of Heart Failure and Transplantation, Rajaie \\ Cardiovascular Medical and Research Center, Iran University of \\ Medical Sciences; Tehran-Iran \\ 'Cardiovascular Research Center, Kerman University of Medical \\ Sciences, Kerman-Iran
}

\section{Introduction}

Heart Failure Association of the European Society of Cardiology defines Takotsubo syndrome as the transient regional wall motion abnormalities occurring in the ventricles, which are incompatible with a single coronary artery distribution apart from new and reversible changes depicted in the electrocardiogram (ECG). It is frequently associated with stressful triggers, considerable elevation of natriuretic peptide, and usually small changes in cardiac troponin. Evidence of ventricular systolic function recovery on cardiac imaging should be present at the follow-up to confirm its diagnosis and treatment (1).

Myocarditis and acute coronary syndrome are the two major differential diagnoses of Takotsubo syndrome.

The objective of this report is to present a case of Takotsubo syndrome with myocarditis or acute coronary syndrome features and without major stress.

\section{Case Report}

A 63-year-old woman presented with pain in her chest and both arms was admitted to the hospital (Ragaie Cardiovascular, Medical and Research Center, Tehran, Iran). The examination of her medical history revealed major depression and thyroiditis. Additionally, she had been taking fluoxetine, clonazepam, and levothyroxine for several years. The pulse rate was 90 per minute and the blood pressure was $160 / 90 \mathrm{~mm} \mathrm{Hg}$. Few minutes later after her admission, she suffered weakness, nausea, vomiting, and a drop in blood pressure to $60 / 30 \mathrm{~mm} \mathrm{Hg}$. The administration of inotrope was started, and she was hospitalized in coronary care unit (CCU). Laboratory findings were unremarkable except for high troponin $(3.83 \mathrm{ng} / \mathrm{ml}$, normal range of $<0.16)$ and pro-brain natriuretic peptide levels $(664.2$, the normal range was less than 125). ECG showed nonspecific ST-T changes. Transthoracic echocardiography demonstrated severe left ventricle (LV) enlargement and dysfunction with left ventricular ejection fraction (LVEF) of approximately $10 \%$, mild right ventricle (RV) enlargement with moderate systolic dysfunction, mild to moderate MR, and severe TR with TRG of approximately $10 \mathrm{~mm} \mathrm{Hg}$. She re- ceived aspirin, statin, inotrope, and heparin. Coronary angiography was normal. LV injection showed wall motion abnormality at apex. About $24 \mathrm{~h}$ later, the blood pressure was increased, leading to the discontinuation of inotrope. Lisinopril, spironolactone, and carvedilol were added to her routine administration of medicines, whereas acute coronary syndrome drugs were discontinued. Cardiac magnetic resonance imaging (MRI) showed a top normal LV size with LVEF approximately $39 \%$, apical ballooning with the preserved contractility of other segments, and normal RV size and function (Video 1). There was no evidence of myocardial delayed enhancement; however, T2 weighted-double inversion recovery images showed prominent diffuse myocardial inflammation (edema) more prominent in apical segments (Fig. 1). This observation led to the suggestion of Takotsubo and myocarditis. After 1 week, the general condition of patient and echocardiographic findings were considerably improved with the near normalization of both ventricle functions. Hence, she was discharged. A follow-up after 2 weeks revealed no signs and symptoms of heart failure. The echocardiography findings were as follows: a normal LV size with global EF approximately $50 \%$, normal RV size, and function without valvular involvement.

The follow-up of patient 1 year later showed no residual signs or symptoms of the disease.

\section{Discussion}

The precise incidence of Takotsubo syndrome is yet to be determined. The absence of a trigger or presence of an emotional

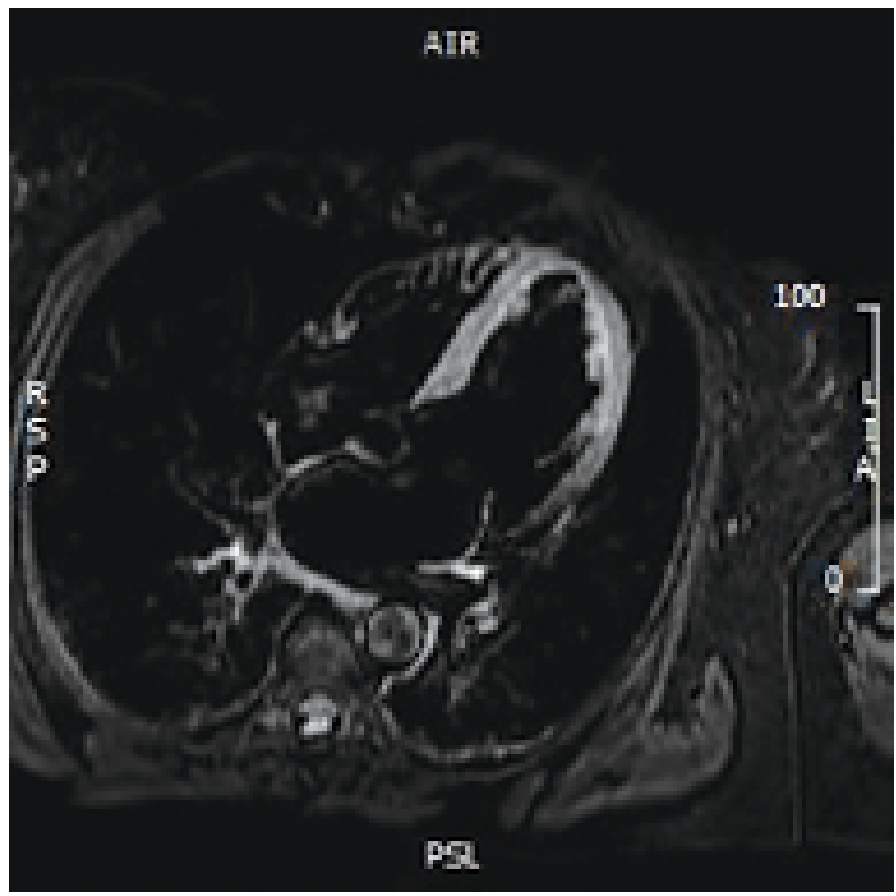

Figure 1. T2 weighted CMR image, showing diffuse myocardial edema, more prominenet in apical segments 
stress as a triggering factor is more common in women. Some points in the patient's history and clinical features could differentiate Takotsubo syndrome from myocarditis: stressful trigger vs. viral illness, females $>50$ year old vs. involvement of young adults, rarely normal ECG changes vs. normal ECG findings in several cases, low or moderate rise in troponin vs. frequent rise in troponin, usually absent LGE in the acute phase vs. non-ischemic late gadolinium enhancement (LGE) pattern (usually subepicardial), usually absent vs. often positive viral genome. These conditions are observed while comparing Takotsubo syndrome and myocarditis, respectively (1).

The unique features of stress cardiomyopathy observed in MRI are myocardial edema and remarkable LV ballooning combined with the absence of significant LGE (2).

Although Takotsubo syndrome typically involves the LV apex, there are atypical forms such as LV basal or RV involvement. Proposed mechanisms are catecholamine hyperactivity, multivessel coronary spasm, microvascular dysregulation, and estrogen deficit. The prominent role of catecholamines in this syndrome increases the possible treatment efficacy of sympathectomy and sympathetic blocks. A lower prevalence of Takotsubo syndrome in the diabetic population supports this theory because of the autonomic dysfunction in diabetics (3).

Takotsubo syndrome is the final diagnosis in approximately $2 \%$ of patients with ACS presentation. Cardiac troponin levels are lower in this syndrome than in myocardial infarction, whereas the BNP levels are higher in this syndrome than in myocardial infarction.

LGE in myocarditis has a patchy distribution, whereas LGE is usually absent in Takotsubo (4). Some reports have shown that the known cardiovascular risk factors may be less obvious in the Takotsubo syndrome, whereas mental or neurologic disorders may be more prominent in the past medical history of patients (5).

\section{Conclusion}

The age and sex of our patient, history of depression, the absence of late gadolinium enhancement in MRI, and complete resolution of disease markers after 2 weeks were in the favor of recovery from Takotsubo syndrome.

Informed consent: The informed consent was obtained from the patient.

Video 1. Steady state free precessions cine image in 4 chamber view shows dyskinesia and apical ballooning with well preserved contractility of basal segments.

\section{References}

1. Lyon AR, Bossone E, Schneider B, Sechtem U, Citro R, Underwood $\mathrm{SR}$, et al. Current state of knowledge on Takotsubo syndrome: a
Position Statement from the Taskforce on Takotsubo Syndrome of the Heart Failure Association of the European Society of Cardiology. Eur J Heart Fail 2016; 18: 8-27. [CrossRef]

2. Eitel I, von Knobelsdorff-Brenkenhoff F, Bernhardt P, Carbone I, Muellerleile K, Aldrovandi A, et al. Clinical characteristics and cardiovascular magnetic resonance findings in stress (takotsubo) cardiomyopathy. JAMA 2011; 306: 277-86. [CrossRef]

3. Gowdar S, Syal S, Chhabra L. Probable protective role of diabetes mellitus in takotsubo cardiomyopathy: a review. Vessel Plus 2017; 1: 129-36. [CrossRef]

4. Scantlebury DC, Prasad A. Diagnosis of Takotsubo cardiomyopathy. Circ J 2014; 78: 2129-39. [CrossRef]

5. Dawson DK. Acute stress-induced (takotsubo) cardiomyopathy. Heart 2018; 104: 96-102. [CrossRef]

Address for Correspondence: Maryam Chenaghlou, MD, Department of Heart Failure and Transplantation, Rajaie Cardiovascular Medical and Research Center, Iran University of Medical Sciences;

Valiasr Ave,

Hashemi Rafsanjani Blvd,

Tehran-Iran

Phone: +09144012182

E-mail: mchenaghlou@yahoo.com

(C) Copyright 2020 by Turkish Society of Cardiology - Available online

at www.anatoljcardiol.com

DOI:10.14744/AnatolJCardiol.2020.45773

\section{Giant right sinus of Valsalva aneurysm led to proximal right coronary artery occlusion}

Ke Wei, (D) Hongwei Guo, (D) Fang Fang, (D) Xiang-yang Qian Department of Vascular Surgery, Fuwai Hospital, National Center for Cardiovascular Diseases, Chinese Academy of Medical Sciences and Peking Union Medical College; Beijing-China

\section{Introduction}

Coronary artery stenosis or occlusion due to sinus of Valsalva aneurysm (SVA) is rare, while SVA leading to right coronary artery occlusion is extremely rare (1-3). We present a case of a giant right SVA combined with proximal right coronary obstruction, wherein good results were achieved in the patient with early surgical intervention.

\section{Case Report}

A 56-year-old male was admitted to our hospital with a 6-month history of shortness of breath and palpitation following physical exertion. Transthoracic echocardiography revealed a giant right SVA. The aortic valve was tricuspid with trivial aortic regurgitation and the ascending and descending aorta were 